\title{
Linx
}

Revue des linguistes de l'université Paris X Nanterre

64-65|2011

Les genres de discours vus par la grammaire

\section{Analyser des écrits promotionnels : prendre en compte les propriétés matérielles de l'espace graphique dans la description grammaticale?}

Julie Lefebvre

\section{OpenEdition}

\section{Journals}

Édition électronique

URL : http://journals.openedition.org/linx/1415

DOI : $10.4000 /$ linx. 1415

ISSN : 2118-9692

Éditeur

Presses universitaires de Paris Nanterre

\section{Édition imprimée}

Date de publication : 1 juillet 2011

Pagination : 207-222

ISSN : 0246-8743

\section{Référence électronique}

Julie Lefebvre, «Analyser des écrits promotionnels : prendre en compte les propriétés matérielles de l'espace graphique dans la description grammaticale? », Linx [En ligne], 64-65 | 2011, mis en ligne le 01 juillet 2014, consulté le 04 mai 2019. URL : http://journals.openedition.org/linx/1415; DOI :

10.4000/linx.1415 


\title{
Analyser des écrits promotionnels : prendre en compte les propriétés matérielles de l'espace graphique dans la description grammaticale?
}

\author{
Julie Lefebvre \\ Université de Lorraine, CREM
}

\section{Introduction}

Nous proposons ici de travailler le croisement du domaine des «genres de discours » avec celui de la « grammaire de la langue » en reliant différents moments du parcours qui a été le nôtre dans le cadre d'une étude linguistique et discursive de réalisations contemporaines (des cinquante dernières années) et imprimées de «notes en bas de page $»^{1}$.

Nous définissons cette forme linguistico-typographique comme un type d'annotation qui se caractérise par l'alliance de deux traits. Ces annotations sont ainsi toutes indexées dans le corps du texte grâce à un signe de typographie-ponctuation traditionnellement qualifié d'«appel de note» et qui prend actuellement le plus souvent la forme d'un chiffre en exposant ou d'un astérisque. Elles se distinguent en outre par leur localisation dans l'espace visuel, qui est donné comme un "ailleurs" relativement à l'espace occupé par le corps du texte, et ce, qu'il s'agisse du bas de la page ou de la fin du volume comme on l'observe le plus fréquemment, de la fin d'une section du volume ou encore, quoique plus rarement, de la marge du corps du texte.

${ }^{1}$ Voir Lefebvre, 2007. 
Nous utiliserons dorénavant l'appellation de "note » pour désigner les annotations reposant sur la mise en œuvre conjointe de ces deux traits.

Il s'agira ainsi dans un premier temps d'évoquer les modalités selon lesquelles se pose la question des genres de discours dans le cadre d'une étude de la note. Dans un second temps, nous exposerons quelques problèmes grammaticaux relatifs à la mise en relation de la note avec le corps du texte dont elle dépend. Nous montrerons, dans un troisième temps, comment la mise en évidence de ces mêmes problèmes grammaticaux est étroitement liée aux genres de discours dans lesquels ils sont observés. Nous conclurons enfin en soulevant quelques questions relatives à la distinction grammaire de l'oral/grammaire de l'écrit.

\section{La question des genres de discours dans l'étude de la note}

Précisons, de façon liminaire, que le terme de « genre » sera ici utilisé, selon les cas, soit dans le sens qui est le sien dans la tradition littéraire, soit dans l'une de ses acceptions en analyse de discours. Par « genre », on comprendra ainsi ce qui permet de « repérer et de classer les différents textes littéraires » en fonction de critères «à la fois de composition, de forme et de contenu» ou encore en fonction de critères « renvoyant à la structure des textes et particulièrement à leur organisation énonciative $»^{2}$. On distinguera de la sorte, par exemple, le roman, l'essai et la biographie. Le terme de "genre» sera sinon employé comme renvoyant à des "événements communicationnels ou à des actes illocutionnaires »3 ainsi, par exemple, du genre de la "vulgarisation scientifique»; ou comme renvoyant à des "grands domaines» (par exemple, "le scientifique») ou à des "micro-domaines d'activités reconnus dans la société »", par exemple le «formulaire administratif».

La question des genres discursifs s'est posée très tôt dans notre travail dans la mesure où, contrairement à ce que l'on croit souvent, la note n'est pas, dans ses réalisations actuelles, une forme réservée aux seuls genres livresques et académiques. S'il est vrai que la note s'observe dans les textes scientifiques et littéraires, elle est en effet également très utilisée dans la presse - spécialisée ou non -, de même que dans des genres qui, liés à d'autres supports que le livre, engagent d'autres modes d'écriture et de lecture que ceux qui sont rattachés à cet objet. Il en va ainsi de tout le champ des écrits promotionnels ou encore des formulaires administratifs, qui recourent massivement au support de la feuille volante et de ses dérivés (prospectus ou cartes postales, par exemple). C'est également le cas de ce que l'on peut qualifier d'«écrits sur emballages» - emballages alimentaires notamment - réalisés sur des supports matériels extrêmement divers tant du point de vue de la nature du volume considéré (parallélépipède, cylindre, ...), que du matériau utilisé (carton, fer, plastique, ...) ou encore de la « stabilité » de la forme de l'objet au cours de son utilisation (présence d'une partie détachable ou non, ...).

Eu égard à l'hétérogénéité des genres discursifs concernés par la note, notre but était double. Il s'agissait tout d'abord de proposer une caractérisation et une description

\footnotetext{
${ }^{2}$ Charaudeau, 2002 : 278.

${ }^{3}$ Branca-Rosoff, $1996: 198$.

${ }^{4}$ Ibid.
} 
générale du fonctionnement linguistique de la note qui soit, précisément, indépendante du fonctionnement de la note dans un genre particulier, en même temps que nous souhaitions amorcer une caractérisation de genres discursifs à partir du fonctionnement de leurs notes. De façon à satisfaire à ce double objectif, nous avons constitué un corpus de mille notes, associées à la partie du corps du texte où s’inscrivait l'appel de note association que nous qualifierons dorénavant de «forme ». Ces mille formes ont été classées en différents secteurs en fonction du genre discursif dont nous les considérions représentatives.

À l'heure où une grande majorité des études linguistiques sont menées sur des corpus construits à partir de bases de données informatisées - telles que, par exemple et pour ce qui concerne les études de l'écrit, la base Frantext -, le caractère «artisanal» de la constitution de notre corpus se justifie par le constat d'une double absence. Ces bases de données, malgré toutes leurs qualités et leur richesse, ne proposent en effet tout d'abord pas un éventail suffisamment large des différents genres représentés à l'écrit - allant par exemple, dans notre cas, de l'essai théorique à l'opercule d'un récipient alimentaire - ; en outre, ne prenant pas en compte la dimension proprement spatiale des faits de langue réalisés à l'écrit, elles ne reproduisent ni leur mise en page ni leur typographie, entravant, voire rendant ainsi impossible leur description, notamment grammaticale, comme nous le verrons plus loin 5 .

Les lignes qui suivent nous permettront de donner un rapide aperçu de la diversité générique du corpus que nous avons élaboré, comme de sa structuration ${ }^{6}$. La première moitié du corpus se compose ainsi de formes observées dans des écrits réalisés sur un support autre que celui du livre. Trois cents formes ont été extraites de textes de presse de périodicité diverse et de contenu varié - soit Libération, Le Figaro, Le Parisien, Le Nouvel Observateur, Charlie Hebdo, L'Équipe, ELLE, les Cahiers du cinéma, Santé magazine et Valeurs mutualistes. Les deux cents autres formes proviennent d'écrits réalisés sur des supports divers - carte postale, ticket, feuille volante, dépliant, carnet, cahier, emballage alimentaire - et sont classées dans trois secteurs distincts du corpus selon qu'ils ont une fonction promotionnelle, une fonction administrative (il s'agit dans ce cas principalement de formulaires) ou qu'ils relèvent du genre «écrits sur emballage ».

La seconde moitié du corpus rassemble quant à elle des formes recueillies sur un seul et même support qui est celui du livre. Une centaine de formes appartiennent à des textes de type instructionnel ou présentant une forte dimension didactique, ainsi, par exemple, des notes relevées dans un recueil de recettes de cuisine, dans un cours de physique à l'usage des étudiants de premier cycle universitaire ou encore dans un

${ }^{5}$ Il semble ainsi qu'on ne dispose pas encore de bases de données suffisamment riches pour permettre une grammaire descriptive des différents usages du français écrit. De ce point de vue, on observe donc une situation similaire à celle dont H.-J. Delofeu faisait le constat (communication orale disponible sur le site du CPTC, université de Bourgogne) relativement à l'absence d'un corpus rendant possible, dans le domaine des études du français parlé, une grammaire des usages de la langue parlée spontanée.

${ }^{6}$ Pour une description plus détaillée du corpus, on se reportera à Lefebvre, 2007. Précisons que la seule restriction que nous ayons apportée à notre relevé a été d'ordre énonciatif : nous avons ainsi choisi de recueillir des structures bilinéaires dans lesquelles la note et le corps du texte se donnent comme réalisées par un même énonciateur et dans un même temps d'énonciation. 
Julie Lefebvre

manuel d'histoire destiné aux élèves des classes de terminale. Cent autres formes ont été identifiées dans des textes présentant une dimension littéraire, tels que, par exemple, $W$ ou le souvenir d'enfance de G. Perec, Notes sur le cinématographe de R. Bresson, ou encore Ombres chinoises de S. Leys. Deux biographies - Jacques Lacan - Esquisse d'une vie, histoire d'un système de pensée d'E. Roudinesco et Le témoignage est un combat - Une biographie de Germaine Tillion de J. Lacouture - ont été à la source d'une nouvelle centaine de formes. Cent formes ont été relevées dans deux ouvrages de synthèse destinés au grand public - L'homme de paroles de C. Hagège et Les hommes - passé, présent, conditionnel d'A. Langaney - et dans une somme historique - Le Proche-Orient éclaté 1956-2003 de G. Corm. Cent dernières formes ont enfin été extraites de deux textes scientifiques - La Distinction de P. Bourdieu et La coénonciation en français - Approches discursive, conversationnelle et syntaxique de T. Jeanneret.

\section{En quoi consiste une approche syntaxique de la note?}

Un des buts de l'étude du fonctionnement général de la note que nous avons menée sur la base du corpus présenté plus haut était de savoir comment, et jusqu'où, il était possible de caractériser, sur le plan syntaxique, l'articulation de la chaîne graphique de la note à la chaîne graphique du corps du texte. Plus exactement, il s'agissait de savoir comment pouvait être décrite la relation de dépendance observée entre tout ou partie de la note et un segment plus ou moins étendu du corps du texte.

Nous avions ainsi à rendre compte, d'un point de vue syntaxique, d'une configuration textuelle "bilinéaire » composée de deux chaînes graphiques qui, situées dans des lieux distincts de l'espace graphique, sont articulées l'une à l'autre autour du signe de typographie-ponctuation double constitué par l'appel de note, inscrit dans le corps du texte, et par son « reflet», le « renvoi de note », qui initie la ligne de la note ${ }^{7}$. Cette configuration, dont l'inscription discontinue dans l'espace graphique apparait comme l'une des propriétés premières, peut être représentée de la façon suivante :

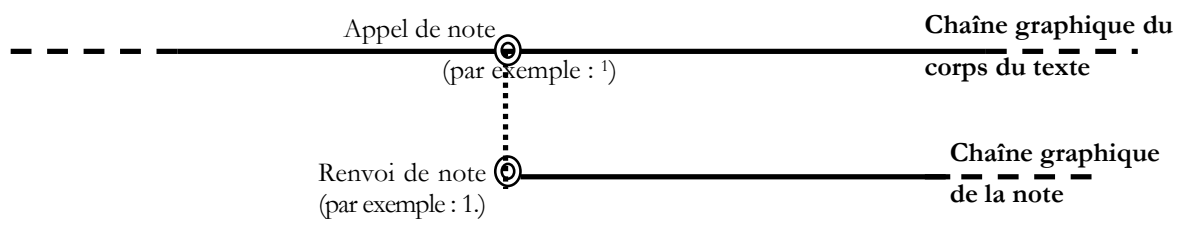

L'étude de notre corpus nous a permis de mettre en évidence trois grands types d'articulation de la note au corps du texte :

- deux types d'articulations qualifiés de «syntaxiques » dans le sens où l'on peut considérer que cette articulation se joue dans le cadre de la phrase, et peut plus ou moins être décrite — on va voir comment — par une syntaxe des catégories ;

\footnotetext{
${ }^{7}$ Sur la notion de "signe de ponctuation double », nous renvoyons à Catach, 1994. Les parenthèses et les tirets doubles font par exemple également partie de cette catégorie.
} 
- un type d'articulation que nous avons identifié comme «textuel» dans la mesure où la relation de dépendance entre la note et le corps du texte n'a alors plus cours dans le cadre d'une phrase, mais se produit entre deux phrases.

Avec une fréquence de $62 \%$ dans notre corpus, ce dernier type d'articulation, textuel, est celui que nous avons observé le plus souvent. Différentes formes de « liage » ${ }^{8}$ interphrastique soutiennent alors la relation entre la note et le corps du texte que ce soit, par exemple, dans la mise en œuvre de phénomènes d'anaphore comme dans les exemples (1) et (2) ci-dessous, ou encore dans le recours à des opérations de connexion, ainsi dans l'exemple $(3)^{9}$ :

(1) La lassitude est telle que la France, l'Allemagne et surtout le nouvel empereur Charles Ier * cherchent à négocier secrètement une paix éventuelle.

$>/<*$ Il succède, en 1916, sur le trône austro-hongrois, au vieil empereur FrançoisJoseph, âgé de 86 ans, et qui règne depuis 1848. [Manuel d'histoire, p. 26/26]

(2) Autre coup de griffe à la mémoire de la série, on apprendra que ce méchant n'est autre que Jim Phelps, soudain ressuscité, dans la scène où Ethan est confronté à Jim et où ce dernier lui explique comment il a été abusé, en faisant semblant de croire que le méchant est Kittrige, l'homme de la CIA, tandis qu'une série d'images mentales qui défilent dans la tête d'Ethan montrent, étape par étape, la reconstitution des meurtres de tous les autres membres de l'équipe par Jim, ainsi que la mise en scène de sa propre mort ${ }^{5}$.

$>/<5$. Ce brillant passage contrapuntique nous rappelle le flash-back traumatique de Geneviève Bujold dans Obsession, où Bujold adulte prend la place de son «moi » enfantin, dans une suite de scènes montrant ce qui s'est vraiment passé la nuit où elle avait été kidnappée. [CdC 505, p. 58/64]

(3) Le 29 octobre, militaires israéliens et égyptiens commencent à discuter à la borne kilométrique 101, devenue depuis célèbre, sur la route du Caire à Suez. La quatrième guerre israélo-arabe est terminée ${ }^{1}$.

$>/<1$. Des combats auront cependant encore lieu sur le Golan syrien. [ProcheOrient, p. 362/362]

Deux cas de figure se présentent lorsque la dépendance de la note au corps du texte peut être qualifiée de «syntaxique» du fait qu'elle se produit dans le cadre d'une seule et même «phrase ». Dans le premier, le moins fréquent $(10 \%$ environ des formes du corpus), on observe des relations de «rection» au sens large de ce terme, dans la

\footnotetext{
${ }^{8}$ Sur cette notion, nous nous référons à Adam, 2006.

${ }^{9}$ Nous proposons une «transcription linéaire » des formes que nous étudions, ne rendant pas compte des caractéristiques de la mise en page d'origine. Nous reviendrons plus bas sur les limites de cette transcription pour l'analyse. Dans les exemples que nous donnons, nous retranscrivons la note dans son intégralité ainsi que la phrase - alors définie typographiquement, telle que délimitée par une majuscule et un point - du corps du texte dans laquelle s'inscrit l'appel de note. Le signe $\|>/<»$ symbolise la mise en relation, à travers l'espace graphique, du signe d'appel de note avec son reflet, le « renvoi de note». On trouvera, à la fin du présent article, la liste des abréviations utilisées dans les références des exemples.
} 
Julie Lefebvre

mesure où on a des constructions rattachées à la catégorie du nom. On peut alors dire que tout (comme en (4)) ou une partie seulement de la note (ainsi en (5) et (6)) est «régi» par un nom qui, localisé en cours ou en fin de phrase, se situe toujours immédiatement avant l'appel de note :

(4) Contrairement à la thalassothérapie ou aux séjours dits de remise en forme ${ }^{(3)}$, le thermalisme a fait ses preuves.

$>/<$ (3) qui ne font appel aux examens médicaux que dans le but de dépister les éventuelles contre-indications aux soins donnés. [ $\mathrm{V} m 219$, p. 25/25]

(5) Ce qui commence par l'obstination de Razieh à acquérir un poisson rouge, alors qu'elle peut en avoir autant qu'elle veut dans le bassin qui se trouve dans sa cour ${ }^{1}$.

$>/<1$. Où un jeune voisin vient en pêcher, qu'il revend semble-t-il clandestinement au vendeur de poissons (mais la scène n'est pas très claire : on ne voit pas bien s'il s'agit du même enfant). [CdC 497, p. 48/48]

(6) Le «Dictionnaire du cinéma »(12) précise ainsi ce gain «/Avec l'orthochromatique/ la transcription des diverses teintes composant un visage, en noir et blanc, restait en déséquilibre. Pour remédier à cet état de choses, les acteurs étaient maquillés en bleu et ocre. [...]. »

$>/<$ 12) De Maurice Bessy et J.-L. Chardans (Pauvert) : le seul où l'on puisse trouver quelques précisions techniques et un peu d'histoire des techniques; l'«Encyclopaedia Universalis », chapitre «Technique d'un cinéma », intitule un paragraphe «émulsions » et n'y traite que du format des films. [CdC 233, p. 44/44]

Dans le second cas de figure, représenté dans 28\% environ des formes du corpus et qui constitue donc le troisième grand type d'articulation entre le corps du texte et la note, si la relation de dépendance se joue toujours dans le cadre d'une phrase, il n'est cependant plus possible de parler de rection, même dans une acception large de la notion. Cette catégorie regroupe tout d'abord les notes qui apparaissent comme des éléments traditionnellement qualifiés de circonstants dans leur relation au corps du texte; leur fréquence est de $5 \%$ dans notre corpus. Il en va ainsi des syntagmes prépositionnels inscrits en note en (7) et (8), et des propositions subordonnées circonstancielles observables en note en (9) et (10) :

(7) Un enfant de six ans sur cinq souffre d'un trouble de la vision, à son entrée à l'école primaire(1).

$>/<$ (1) Selon une enquête réalisée en 1999-2000 sur plus de 30000 élèves à partir des examens de santé à l'entrée en primaire, par la Caisse primaire d'assurance maladie de Paris. [Vm 221, p. 26/27]

(8) Quel que soit l'endroit d'où vous appelez en France métropolitaine, le prix de l'appel est le même*.

$>/<*$ Hors appels depuis un mobile ou avec une carte France Télécom. [Écrits promotionnels, France Télécom] 
(9) Par aucun moyen un $\mathrm{K}_{0}$ ne pourra produire une particule $\Delta$ lorsqu'il interagit avec la matière ordinaire (des protons et des neutrons).*

$>/<*$ Sauf, bien sûr, s'il produit aussi deux $\mathrm{K}^{+}$ou d'autres particules d'étrangeté totale +2 . Il suffit d'imaginer qu'il s'agit ici de réactions où l'énergie est insuffisante pour produire ces particules supplémentaires étranges. [Cours de physique, p. 219/219]

(10) Il faut d'ores et déjà savoir et réaffirmer que c'est en dehors et contre ces lieux de résistance obscurantiste qu'un tel film est appelé à pénétrer dans le champ d'une plus large lisibilité (1).

$>/<$ (1) Sans tomber dans l'illusion charismatique du regard pur, de l'œil neuf et du don, en espérant une lisibilité instantanée et miraculeuse d'Othon pour les couches sociales les plus défavorisées. Les travaux scientifiques de Bourdieu et Passeron sur la sociologie de la perception esthétique $[. .$.$] ont démontré que$ les ouvriers, devant un produit artistique, ne se situaient pas par rapport à quelque «spontanéité » mais par rapport à leur culture bourgeoise absente [...]. [CdC 224, p. 43/47]

D'autre part, cette même catégorie, qu'on peut définir comme «syntaxique non-rectionnelle », rassemble des formes du type des exemples (11) à (15) :

(11) C'est pourquoi on assortira de quelque pondération le point de vue des biologistes : «Il parait probable (mais évidemment toujours hypothétique) que le développement du lien social, qui prend une grande ampleur chez les primates supérieurs, soit au départ la conséquence et non la cause de l'épanouissement du néo-cortex. $\|^{8}$

$>/<$ 8. J.-P. Changeux, L'homme neuronal, Paris, Fayard, Coll. «Le temps des sciences », 1983, p. 355. [CH, p. 21/21]

(12) Avec ces chèques-cadeaux Fnac, ne résistez pas à la tentation et succombez à vos envies* : disques, cassettes, CD-ROM, cassettes vidéo, appareils photo, téléviseur, HI-FI, camescope, téléphonie, micro-informatique...

$>/<*$ Modalités d'acceptation spécifiques selon les enseignes. [Écrits promotionnels, Télérama, lettre]

(13) Berlin $983 \mathrm{f} *$

$>/<*$ jeunes \& étudiants (billets flexibles) [Écrits promotionnels, Usit, Libération, 28 mars 1999, p. 24/24]

(14) Vous avez juste besoin de composer le 015353 7000** devant le numéro de votre correspondant.

$>/<* *$ Coût d'un appel vers Paris. Valable aussi depuis et vers les téléphones portables. Les tarifs sont valables le jour de leur publication. [Écrits promotionnels, One.Tel, Libération, 22 février 1999]

(15) Téléviseur + magnétoscope + radio-réveil Gratuit!*

$>/<*$ Tirage au sort de 10 gagnants parmi les réponses reçues sous 8 jours. Voir l'extrait de règlement à la fin de la lettre jointe. [Écrits promotionnels, Philips, lettre] 
Julie Lefebvre

Dans les notes des formes du type de l'exemple (11), on relève ainsi une juxtaposition de syntagmes nominaux qui, ensemble, constituent ce que l'on reconnait comme une référence bibliographique. Les notes des formes du type des exemples (12) à (15) constituent un ensemble plus hétérogène ; leur «tête » - où se joue le plus fortement la dépendance de la note au corps du texte ${ }^{10}$ - est néanmoins toujours un syntagme nominal, qui apparaît soit seul (ainsi en (12)), soit suivi de syntagmes de nature diverse - par exemple, syntagme nominal (cf. (13)), syntagme adjectival $(c f .(14))$ - et/ou d'une ou de plusieurs phrases, comme c'est le cas en (15).

Ces formes, dont la fréquence est de $23 \%$ dans notre corpus, ont pour propriété de ne pas être «monolinéarisables». Ceci revient à dire que la succession des éléments de la note et du corps du texte entre lesquels s'observe une relation de dépendance ne pourrait se produire sur une seule et même ligne graphique sans qu'il soit nécessaire d'introduire un "décrochement typographique » ${ }^{11}$ fort (mise entre parenthèses ou entre tirets doubles), un décrochement typographique plus faible (avec le recours à une virgule double ${ }^{12}$, par exemple) ne suffisant en effet pas à rendre acceptables les formes monolinéaires obtenues. C'est ce qu'on peut voir dans les manipulations (11'), (11') et (12'), (12') suivantes $^{13}$ :

(11') C'est pourquoi on assortira de quelque pondération le point de vue des biologistes : «Il paraît probable (mais évidemment toujours hypothétique) que le développement du lien social, qui prend une grande ampleur chez les primates supérieurs, soit au départ la conséquence et non la cause de l'épanouissement du néo-cortex » (J.-P. Changeux, L'homme neuronal, Paris, Fayard, Coll. « Le temps des sciences », 1983, p. 355).

*(11”) C'est pourquoi on assortira de quelque pondération le point de vue des biologistes : «Il paraît probable (mais évidemment toujours hypothétique) que le développement du lien social, qui prend une grande ampleur chez les primates supérieurs, soit au départ la conséquence et non la cause de l'épanouissement du néo-cortex », J.-P. Changeux, L'homme neuronal, Paris, Fayard, Coll. « Le temps des sciences », 1983, p. 355.

(12') Avec ces chèques-cadeaux Fnac, ne résistez pas à la tentation et succombez à vos envies - modalités d'acceptation spécifiques selon les enseignes - : disques, cassettes, CD-ROM, cassettes vidéo, appareils photo, téléviseur, HIFI, camescope, téléphonie, micro-informatique...

*(12") Avec ces chèques-cadeaux Fnac, ne résistez pas à la tentation et succombez à vos envies, modalités d'acceptation spécifiques selon les enseignes : disques, cassettes, CD-ROM, cassettes vidéo, appareils photo, téléviseur, HI-FI, camescope, téléphonie, micro-informatique...

\footnotetext{
${ }^{10}$ Sur les questions relatives à la détermination de différentes «zones » dans la note en fonction du degré de dépendance au corps du texte, nous renvoyons à Lefebvre, 2007.

${ }^{11}$ Sur cette notion, voir Pétillon-Boucheron, 2003.

12 Sur la notion de « virgule double », voir Catach, 1994.

${ }^{13}$ L'astérisque marque la non acceptabilité de la forme obtenue.
} 
Il est important de noter que la «monolinéarisation » de l'ensemble des cas de relation syntaxique entre la note et le corps du texte que nous avons évoqués jusqu'à présent (exemples (4) à (10)) n'implique en revanche pas le recours à des décrochements typographiques forts, la seule introduction de virgules doubles rendant acceptables les formes monolinéaires résultant de la manipulation, ainsi qu'en témoignent les formes $\left(4^{\prime}\right)$ et $\left(9^{\prime}\right)$ :

(4') Contrairement à la thalassothérapie ou aux séjours dits de remise en forme, qui ne font appel aux examens médicaux que dans le but de dépister les éventuelles contre-indications aux soins donnés, le thermalisme a fait ses preuves.

(9) Par aucun moyen un $K_{0}$ ne pourra produire une particule $\Delta$ lorsqu'il interagit avec la matière ordinaire (des protons et des neutrons), sauf, bien sûr, s'il produit aussi deux $\mathrm{K}^{+}$ou d'autres particules d'étrangeté totale +2 .

Les tests effectués précédemment permettent de mettre en évidence le rapport complexe que les formes du type des exemples (11) à (15) entretiennent avec le « caractère linéaire du signifiant», principe dont on connait la centralité dans la définition du signe linguistique. S’il serait ainsi intéressant de montrer que, dans ces formes, la complexité du rapport à la linéarité est à rattacher à la mise en œuvre de procédés relevant de la parataxe, nous nous contenterons de souligner ici le rôle central que jouent les ressources matérielles de l'espace scripturaire dans l'organisation syntaxique de ces formes. Les jeux sur la distance plus ou moins grande qui sépare la note du corps du texte dans l'espace visuel, leur disposition réciproque dans un espace graphique dont la fragmentation apparaît comme l'un des caractères majeurs, ou encore les contrastes de taille, de style et de couleur des caractères respectifs de la note et du corps du texte semblent ainsi assurer la «tenue » syntaxique de ces formes ${ }^{14}$. C'est ce rôle dévolu à la spatialisation et aux jeux typographiques qui est rendu saillant par l'étude des notes dans certains genres discursifs, celui des écrits promotionnels notamment.

\section{Exemples de points d'intersection entre domaines des « genres de discours " et de la " grammaire de la langue»}

Les trois types d'articulation entre la note et le corps du texte que nous venons de présenter ne se répartissent pas de façon homogène dans les différents secteurs de notre corpus, c'est-à-dire selon les différents genres discursifs qui y sont représentés.

Les formes «non-monolinéarisables » du type des exemples (12) à (15) mentionnés plus haut, où l'élément de la note dépendant le plus fortement du corps du texte est un syntagme nominal (et non une séquence de référence bibliographique), apparaissent ainsi avec une fréquence élevée dans le secteur rassemblant les formes extraites d'écrits promotionnels, soit avec une fréquence de 35,55\%, pour une fréquence moyenne de ce type d'articulation dans le corpus de 9,31\%. Il est intéressant de noter que la fréquence de ce type d'articulation est plus faible dans d'autres secteurs

14 On trouvera la reproduction des mises en page originales des exemples (12) à (15) à la fin du présent article. 
du corpus tels que ceux regroupant les formes relevées dans des formulaires administratifs et sur des emballages (fréquences de, respectivement, $18 \%$ et $19 \%$ ), et proche de zéro ou nulle pour les secteurs dont les formes sont issues de livres. Ces résultats statistiques nous amènent à formuler deux séries de remarques, la première - que nous ne développerons pas ici - relative à la caractérisation syntaxique de genres discursifs, la seconde portant sur l'intérêt de la prise en considération de la question des genres discursifs dans l'abord des faits grammaticaux.

Ce type d'articulation syntaxique "non-monolinéarisable » peut tout d'abord être mis au nombre des traits caractéristiques du genre des écrits promotionnels. Il apparait également comme l'une des spécificités syntaxiques des genres discursifs où il est fait recours de façon extrême aux possibilités qu'offre à la langue son inscription dans l'espace graphique. De cette manière, la description des différents types d'articulation de la note au corps du texte participe à un travail de différenciation plus large des genres discursifs mené dans l'attention aux faits grammaticaux.

Il nous semble ensuite que l'étude de ce type d'articulation syntaxique, rendue possible par les recherches effectuées sur un corpus représentatif de différents genres discursifs - dont la variété dépend fortement, en l'occurrence, de la nature des différents supports d'écriture considérés -, témoigne de l'importance de la prise en compte de la question des genres discursifs dans la description des faits grammaticaux. Un travail portant sur l'articulation syntaxique de la note au corps du texte dans les seuls genres discursifs rattachés à l'objet-livre n'aurait en effet pas permis de montrer - ou, du moins, pas de façon aussi nette et aussi massive - la nécessité d'inclure, dans l'étude des faits grammaticaux écrits, l'attention à leurs caractéristiques proprement matérielles, susceptibles d'occuper une place de premier plan dans la description et, dans tous les cas, jamais anodines.

De ce point de vue, il nous semble qu'il serait intéressant de prolonger les travaux de J. Gardes-Tamine (2004) portant sur l'élaboration d'une «grammaire de l'écrit » - travaux menés sur un corpus d'écrits réalisés dans des livres, et à dimension littéraire - à partir d'un corpus plus large, incluant également des écrits non littéraires et relevant de genres discursifs non liés à l'objet-livre. Il s'agirait par là de prendre toute la mesure du constat suivant de J. Hébrard : « [1]'écriture est du langage contraint à la spatialisation : il faut tirer toutes les conséquences de cette "contrainte" »" 1 . Ainsi, dans les formes du type des exemples (12) à (15) évoqués plus haut, la description syntaxique de l'articulation note-corps du texte requiert l'attention à des paramètres tels que la taille des caractères dans lesquels sont réalisés, respectivement, le corps du texte et la note, ou encore la disposition que ces deux «blocs» textuels occupent l'un par rapport à l'autre dans l'espace visuel. L'analyse n'étant alors plus possible à partir de la seule « transcription linéaire » des formes (comme cela était en revanche le cas pour les formes du type des exemples (1) à (10)), il convient de travailler directement à partir de leur mise en page d'origine. On observe ainsi une démarche similaire à celle qui a cours dans le champ des études de l'oral, qui se doivent d'élaborer leurs descriptions à partir de transcriptions rendant compte au mieux des différentes dimensions de la langue parlée ${ }^{16}$.

15 Hébrard, 1983, p. 70.

16 Voir Blanche-Benveniste, 1997. 
Analyser des écrits promotionnels ...

\section{Conclusion}

Au-delà de la similitude ponctuelle que nous venons de signaler, une analogie plus générale nous semble à l'œuvre entre, d'une part, le champ des études du français parlé et, d'autre part, celui des études du français écrit tel que nous l'envisageons comme prenant en compte l'extrême diversité des genres de discours à travers lesquels il se réalise, et comme ne faisant pas abstraction, dans l'analyse, des propriétés matérielles du scripturaire. Nous souhaitons, en guise de conclusion, soulever quelques questions à ce sujet, qui ouvriront sur la problématique plus vaste de la distinction entre une grammaire de l'oral et une grammaire de l'écrit.

On aura remarqué que le type d'articulation «non monolinéarisable» entre le corps du texte et la note sur lequel nous avons insisté ici échappe au secteur d'étude délimité par les outils et le cadre d'analyse que nous avions initialement choisis, à savoir ceux, traditionnels, d'une syntaxe de la phrase et des catégories. On se trouve ainsi devant un problème du même ordre que celui qui se pose aux descripteurs de l'oral qui, confrontés à l'insuffisance d'une syntaxe de la phrase et des catégories dans la description se trouvent dans la nécessité de penser un autre cadre théorique pour l'analyse ${ }^{17}$. Il est d'ailleurs intéressant de remarquer que les écrits promotionnels, les écrits sur emballage ou encore les formulaires administratifs - genres discursifs écrits faisant intervenir les propriétés matérielles de l'espace scripturaire dans leur fonctionnement grammatical-présentent une fréquence élevée de formes dans lesquelles la note peut être analysée comme un «circonstant» par rapport au corps du texte, soit comme un «élément périphérique » dont la description nécessite la mise en œuvre d'une tout autre syntaxe qu'une syntaxe de rection.

La question se pose donc de savoir s'il serait possible de reprendre ou, à tout le moins, d'adapter, les modèles d'analyse mis en place dans le champ des études grammaticales de l'oral - nous pensons notamment aux travaux de C. BlancheBenveniste et du GARS - à une description de ces formes extraites de genres typiquement écrits. Telle est l'hypothèse de travail que nous souhaiterions éprouver. Dans le cas où une description macro-syntaxique de ce type de formes se révélait possible, la position d'A. Berrendonner (2004) selon qui il n'y aurait « qu'une grammaire du français, mais des différences d'opportunité pragmatique et cognitive entre ses structures, selon qu'on s'en sert à l'écrit ou à l'oral » ${ }^{18}$ serait confortée.

\footnotetext{
${ }^{17}$ Voir Blanche-Benveniste et al., 1990.
}

18 Berrendonner, 2004 : 250. 
Reproduction de la mise en page des exemples (12) à (15)

(12)

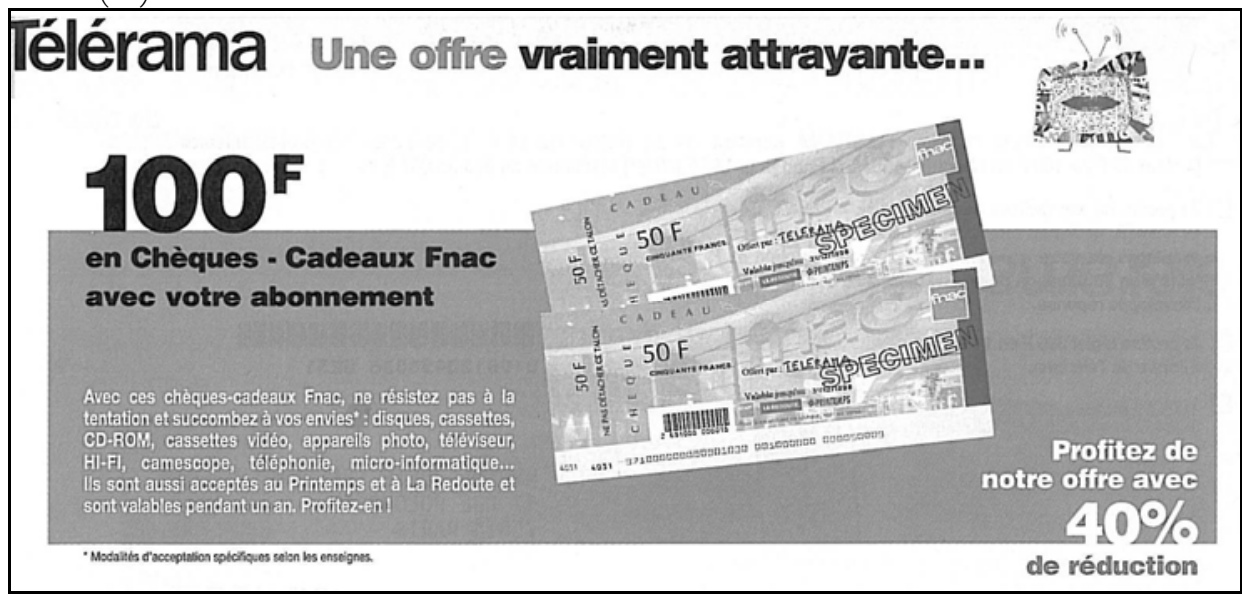

(13)

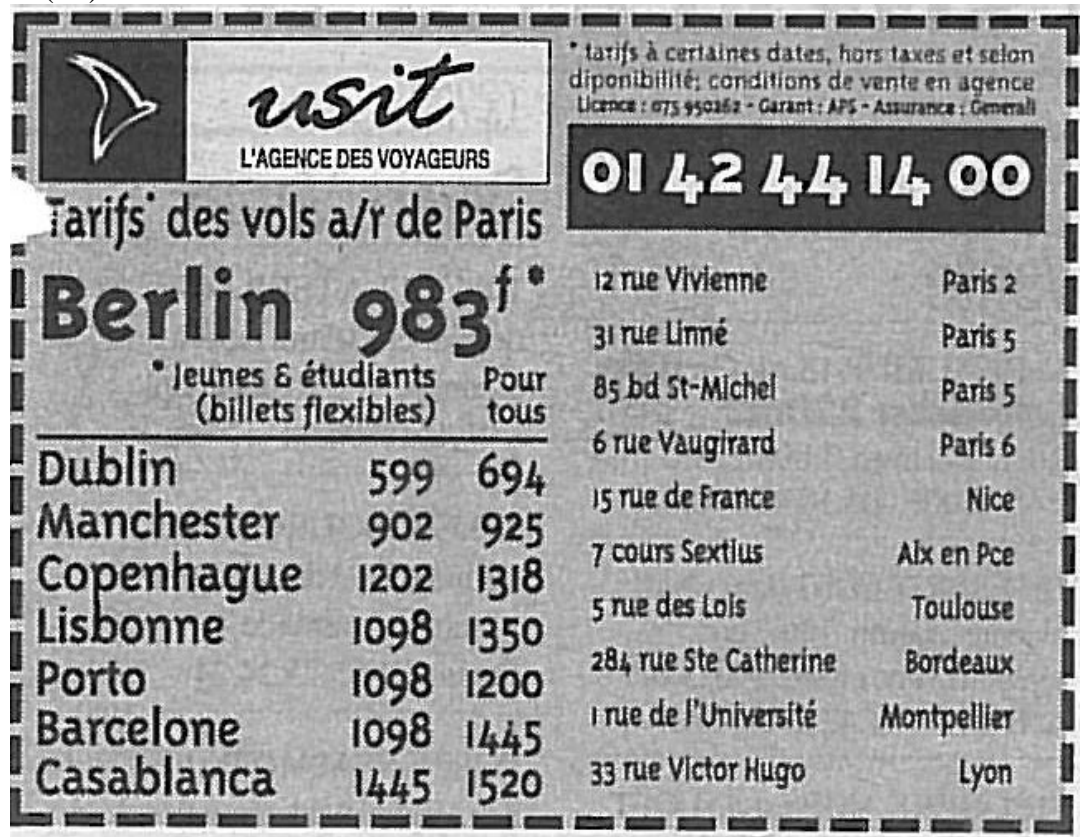


(14)
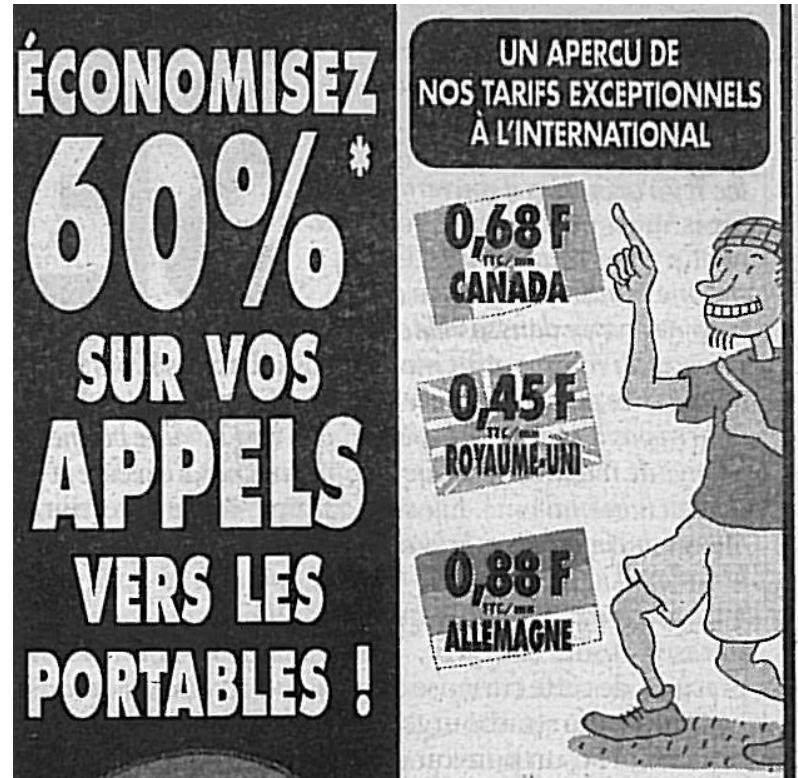

Avec One.Tel, économisez sur tous vos appels : internationaux, nationalux et d'un téléphone fixe vers un téléphone portible. Aucun abonnement, aucune installation, aucune consommation minimum, aucun paiement anticipé. Vous avez juste besoin de composer le $0153537000^{* 2}$ devant le numéro de votre correspondant.

VOS APPILIS A PRIX RENSEIGNEZ-VOUS AU $\sqrt{0 \text { स2140801127 127 }}$ IRRÉESTSUIBLES 24 heures/24 One.Tel ») $100 \%$ Télécoms.

7 jours/7 
(15)

\section{POUR TOUS LES GAGNANTS CE COMBI TV/MAGNÉTOSCOPE/RADIO-RÉVEIL}

Ce combiné TV/magnétoscope/radio-réveil vous surprendra par sa simplicité d'utilisation et d'installation. Idéal lorsqu'on emménage ou en vacances : un seul branchement suffit, la recherche des chaînes se fait automatiquement, et, comble du raffinement, pour une installation simplifiée, la marche à suivre s'affiche à l'écran!

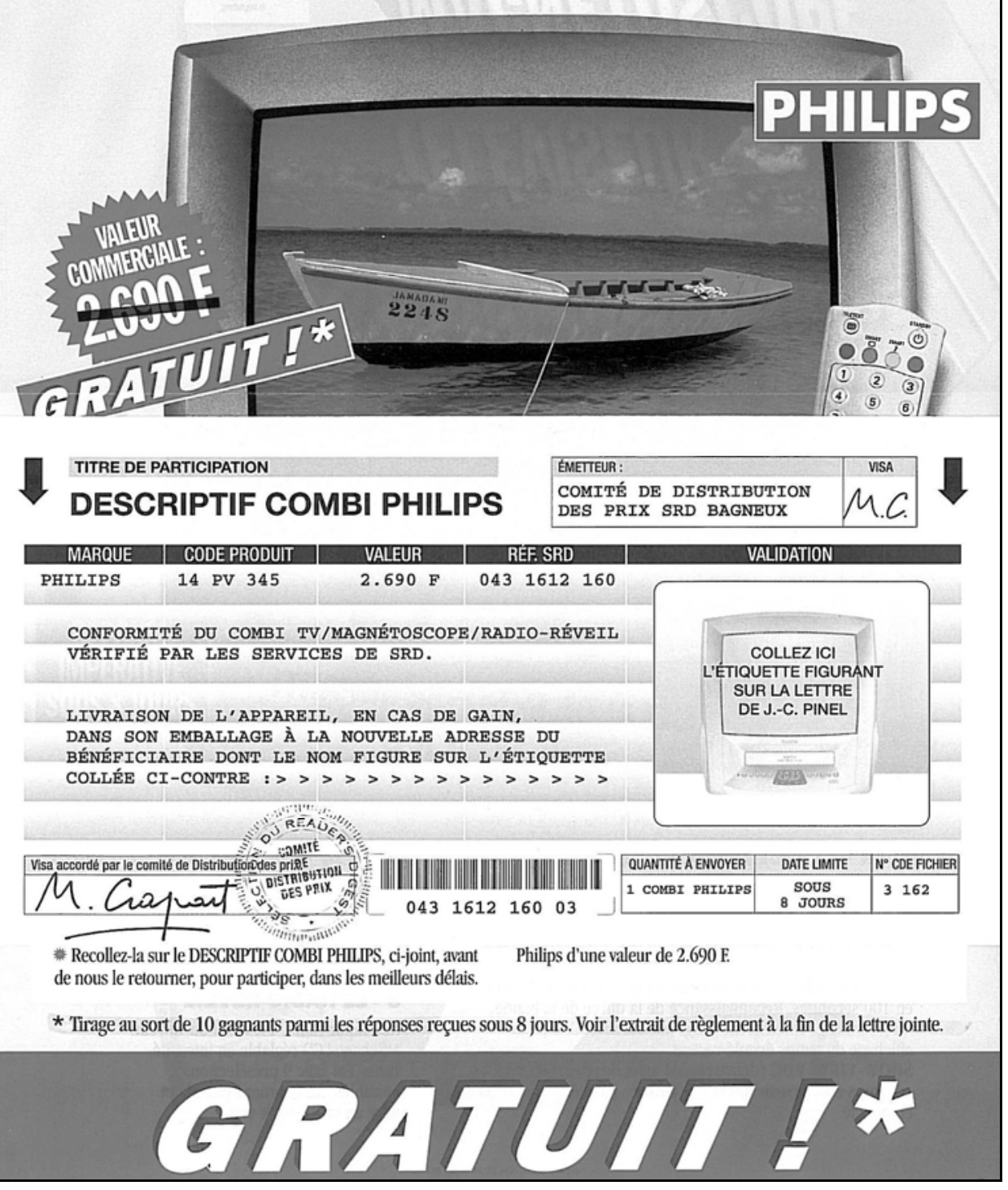




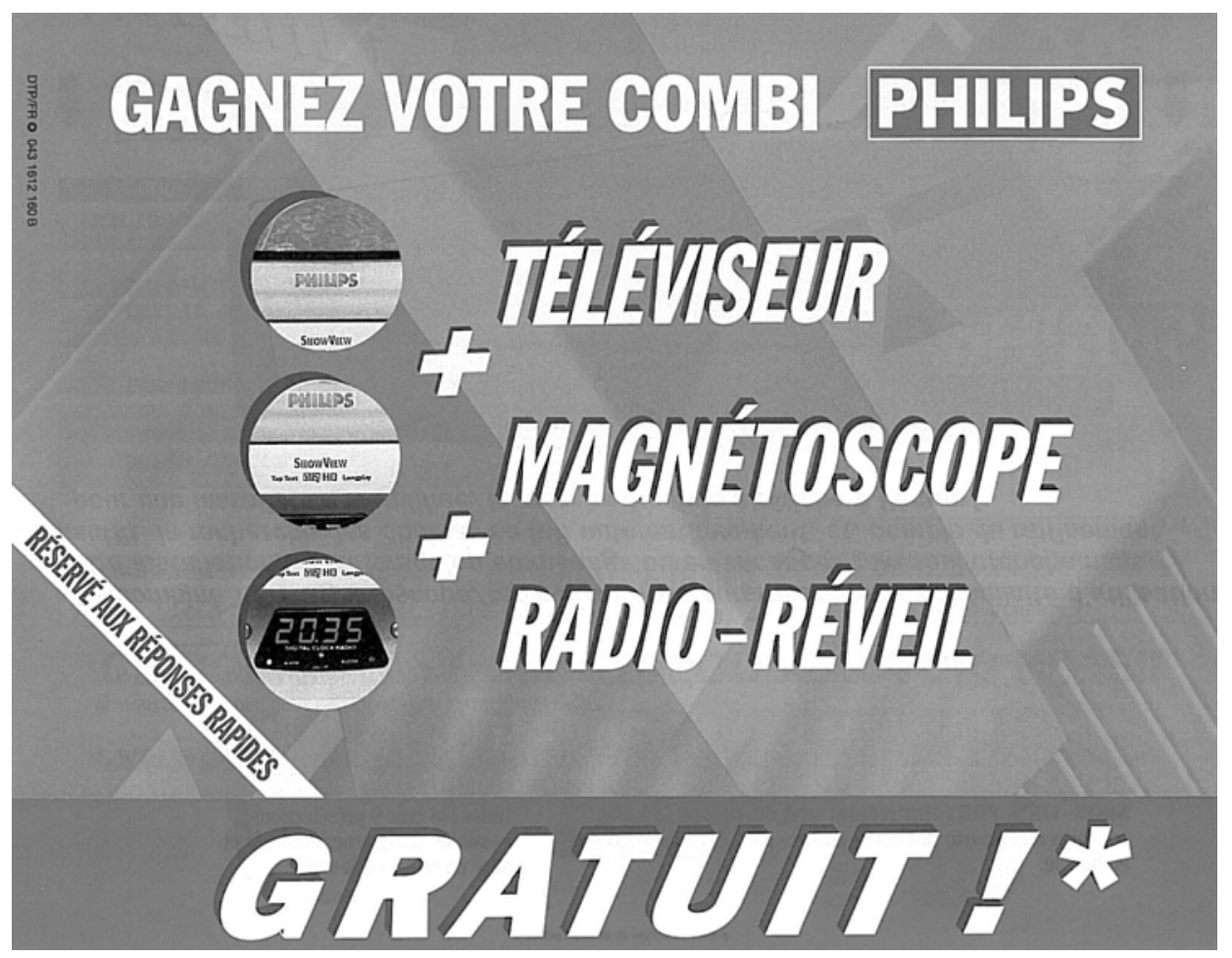

Liste des abréviations utilisées dans les références des exemples

CdC: Cabiers du cinéma $\mathrm{n}^{\circ} 224$ (octobre 1970), n²33 (novembre 1971), nº497 (décembre 1995), n505 (septembre 1996).

CH : Hagège C., 1985, L'homme de paroles, Paris, Fayard.

Cours de physique : Feynman R., Leighton R., Sands M., 1979 (version française de Equer B. et Fleury P.), Le Cours de physique de Feynman, t. 3 ("Mécanique quantique »), Paris, InterEditions.

Manuel d'histoire: Bouillon J., Sohn A.-M. et Brunel F., 1980, Histoire : le monde contemporain 1914/1945, Paris, Bordas.

Proche-Orient: Corm G., 2003, Le Proche-Orient éclaté 1956-2003, Paris, Gallimard, coll. « Folio histoire ».

$\boldsymbol{V m}$ : Valeurs mutualistes $\mathrm{n}^{\circ} 219$ (juillet 2002), nº 221 (novembre 2002). 
Julie Lefebvre

\section{Références bibliographiques}

ADAM J.-M., 2006, La linguistique textuelle - Introduction à l'analyse textuelle des discours, Paris, Armand Colin.

BERRENDONNER A., 2004, "Grammaire de l'écrit vs grammaire de l'oral: le jeu des composantes micro- et macro-syntaxiques» in Rabatel A. (éd), Interactions orales en contexte didactique, Lyon, PUL, p. 249-264.

Blanche-Benveniste C., Bulger M., Rouget C., Van Den Eynde K., 1990, Le français parlé : études grammaticales, Paris, Éditions du C.N.R.S.

Blanche-Benveniste C., 1997, Approches de la langue parlée en français, Paris, Ophrys.

BrancA-Rosoff S., 1996, «Retour aux genres » in Auroux S., Delessalle S. et Meschonnic H. (éds), Histoire et grammaire du sens, Paris, Armand Colin, p. 189-203.

CATACH N., 1994, La ponctuation, Paris, P.U.F., coll. «Que sais-je ?».

Charaudeau P., 2002, "Genre de discours» in Charaudeau P. et Maingueneau D. (éds), Dictionnaire d'analyse du discours, Paris, Seuil, p. 277-281.

Gardes-Tamine J., 2004, Pour une grammaire de l'écrit, Paris, Belin.

HÉBRARD J., 1983, «L'évolution de l'espace graphique d'un manuel scolaire : le "Despautère" de 1512 à $1759 »$, Langue française, n 59, p. 68-87.

LEFEBVRE J., 2007, La note comme greffe typographique: étude linguistique et discursive, Université Paris III - Sorbonne nouvelle, 645 p.

PÉtillon-Boucheron S., 2003, Les détours de la langue : étude sur la parenthèse et le tiret double, Paris-Leuven, Peeters. 